\title{
SIMULATION AND VISUAL CONTROL OF CHIP SIZE FOR CONSTANT SURFACE ROUGHNESS
}

\author{
Zuperl, U. \& Cus, F. \\ University of Maribor, Faculty of Mechanical Engineering, Smetanova 17, SI-2000 Maribor, Slovenia \\ E-Mail: uros.zuperl@um.si,franc.cus@um.si
}

\begin{abstract}
A visual cutting chip control system is designed to automatically adjust feed rate in order to maintain constant surface roughness in ball-end milling. The proposed visual control system has a modular structure, consisting of an optical vision system (OVS), an adaptive cutting chip size-control loop for a feed servo and a surface roughness in-process prediction model. The OVS is employed to acquire the cutting chip sizes form the camera. A division controller is used to control the chip size by modifying the feed rate and consequently maintaining surface roughness constant. Surface roughness is predicted based on the detected chip size. The efficiency of the chip control strategy is tested by series of simulation with various step changes in the cutter/workpiece contact area. For simulation purposes an experimentally validated milling plant simulator with an adopted feed servo drive model and a cutting chip size model is employed. An adaptive neural inference system (ANFIS) is established to effectively simulate the cutting chip size in ball end-milling. In simulation, the reference chip size and consequently the reference surface roughness are well maintained when the cutting-depth profile of a workpiece is varying step-wise or continuously.

(Received in September 2014, accepted in March 2015. This paper was with the authors 2 months for 1 revision.)
\end{abstract}

Key Words: $\quad$ Ball-End Milling, Chip Size, Visual Control, Surface Roughness, Simulation, ANFIS

\section{INTRODUCTION}

Surface roughness is a main factor in monitoring the surface quality of a machined part in wide spread end milling processes. Surface monitoring is usually carried out by manually examining the machined surfaces by using surface stylus devices. According to Al-Kindi and Shirinzadeh [1], this procedure is time consuming, labour intensive and inaccurate due to disturbances from the surroundings.

To assure that the surface roughness of a machined part is within the defined tolerances, an in-process measuring system must be implemented. The output of the in-process measuring system is feedback to adaptive control, which is used to maintain the reference surface roughness. In the past some in-process measuring systems have been developed to determine the surface roughness of the machined piece. In optical systems [2], surface roughness is predicted according to the determined relation between surface image properties and surface roughness. One system uses fibre-optics to measures the diffuseness of the reflected light from the surface [3]. Another system employs a machine vision system to view the machined surface and send the digital data to computer for analysis [4]. Kamguem et al. [5] demonstrated that roughness parameters $\left(R_{a}, R_{t}\right.$ and $\left.R_{z}\right)$ can be estimated by using only image-extracted features. Lu and Tian [6] used light scattering techniques to realize the online prediction of machined surface roughness. Two non-optical methods were also developed. They are based on an inductance pickup and a capacitance probe [7]. Due to disturbances from the fierce machining environment the accuracy of these systems is not sufficient [7]. For milling process, only one applicable non-contact technique to measure roughness in-process has been reported [8]. It employs an acoustic-based ultrasonic sensor for surface monitoring in face milling. The surface roughness is measured by sensing the intensity of ultrasonic beams reflected from the machined surface. Unfortunately, this technique was also found to 
be unsuitable for its in-process use in the production environment due to the inconsistent accuracy and an expensive sensor [9].

In recent years, the research efforts to increase quality of machined parts are directed towards developing of adaptive control systems which contain reliable in-process models [10] that can precisely predict surface roughness. An adaptive control with the on-line surface roughness prediction model could be capable of controlling the surface roughness in real time [11]. Some systems with designed mechanism to control the surface roughness have been developed for turning operations [12, 13], with only a few for milling operations [14].

Due to end milling process complexity, the surface roughness is very difficult to model [15]. This is the main reason why all the research efforts for developing a reliable and applicable model based adaptive control system failed.

Lately, research efforts are focused to the development of indirect surface roughness monitoring systems. Huang et al. [16] presented the application of a neural network algorithm in an in-process Pokayoke system to perform the adaptive surface roughness control in end milling operations. Kirby et al. [17] developed an in-process surface roughness adaptive control system for a turning operation, using a fuzzy-nets modelling and tool vibrations measured with an accelerometer. Zuperl and Cus [18] employed in his model reference adaptive control system (MRAC) a dynamometer as an in-process sensor to indirectly control the surface roughness. The same technique was also applied to the turning process [19].

In order to replace the expensive dynamometer, a novel visual control of cutting chip size is proposed in this research. The cutting chip size control was adopted to maintain surface roughness constant in order to increase the quality of end-milling process. No research efforts have been dedicated so far to develop methodologies for controlling of the cutting chip size in milling. The control strategy capability is demonstrated by simulation with various step changes in the cutter/workpiece contact area. For simulation purposes the experimentally validated milling plant simulator with the surface roughness prediction model is employed. By combining divisional control model and milling plant simulator, a novel chip size control simulation of ball-end milling is developed.

The rest of the paper is organized as follows. The proposed visual chip size control system is described in Section 2. Section 3 covers the chip size control simulator of ball-end milling and the simulation set-up. Finally, sections 4 and 5 present simulation results and conclusion.

\section{VISUAL CONTROL OF CUTTING CHIP SIZE}

The basic idea of this research is to create a visual control as an observation technique of the cutting chip geometry. The objective of the proposed control is therefore to adjust the feed rates and maintain the cutting chip size constant in order to achieve the desired value of the machined surface finish. Fig. 1 shows the basic structure of the chip size control. The proposed system consists of five main parts. The first part is a reference model (Block B1). Its input is the desired surface roughness $R a_{r e f}$ and the output is the desired chip size CSref. The model is developed from a set of data obtained during actual machining tests performed on a Heller milling machine using surface roughness tester and the vision system.

The second part of the proposed system is the milling machine with a Fagor computer numerical control (CNC) unit. The milling machine is combined with the optical vision system (part 3) for assessing the chips size. The output of the optical vision system together with the desired chip size is sent to the system controller (part 4). The measured chip size signals are used in the controller to indirectly regulate the surface roughness $(R a)$. The controller adjusts the feed rate by assigning an override percentage to the CNC controller based on the measured chips sizes. The feed rate command $f_{c}$ is the product of the feed rate override percentage command $f_{c \%}$ and the pre-programmed feed rate. 


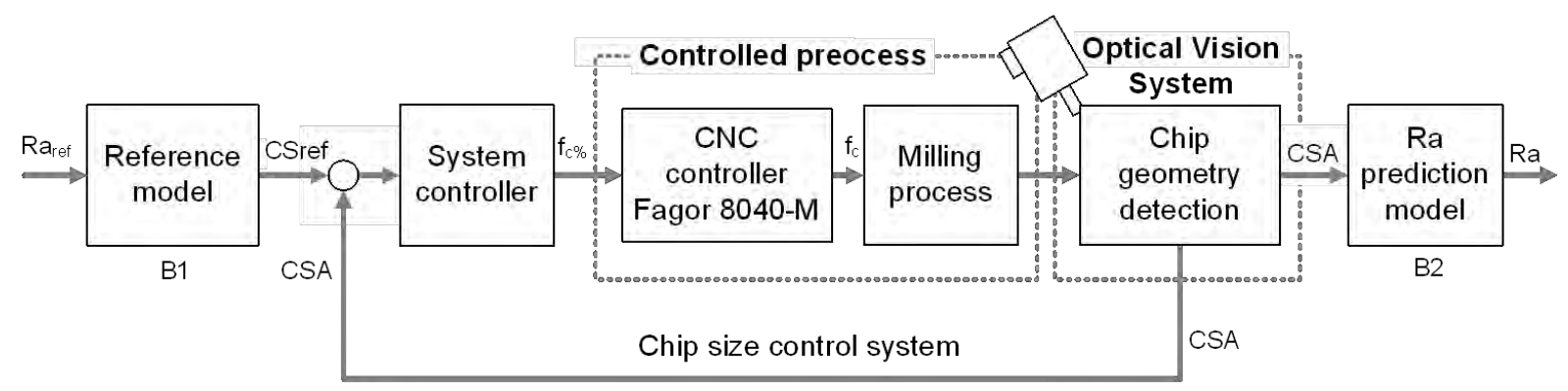

Figure 1: The cutting chip size control structure.

Finally, the last part of the system is a B2 block. This block predicts in-process the surface roughness $\left(R_{a}\right)$ of the machined surface based on measured chip size signals.

The following algorithm is developed to attain the $R a_{\text {ref }}$ by controlling the chip size:

1. The $R a_{\text {ref }}$ is initiated,

2. Based on desired $R a_{r e f}$ the reference chip size CSref is determined according to the block B1,

3. When the CSref is known, the feed rate override command value $f_{c \%}$ is determined,

4. The chip size detection and analysis is performed by the optical vision system; chip size running average $(C S A)$ is calculated,

5. The CSA is compared with the CSref and the quotient is sent to the system controller,

6. Based on the measured chip size the actual $R a$ is determined and displayed on the control panel according to the block B2,

7. The controller adjusts the feed rates and sends the new command $f_{c \%}$ back to the $\mathrm{CNC}$ control unit,

8. Steps 4 to 8 are repeated until the end of machining.

The flow chart of the OVS algorithm is shown in Fig. 2.

\subsection{Chip size detection}

The developed OVS is adapted to milling and a special program was developed to acquire the cutting chip geometry. The program for the detection of recorded chip images was designed in Vision Builder (VB). The developed program is executed in 8 steps: image acquisition, image calibration, image preparation, image tresholding, image filtering, object detection, variable setting (number of chips, chip size) and image displaying.

The simulated method of image capturing is defined in Step 1 (Image acquisition). The settings for the measurement of the chip geometry are set in Step 2 (Image calibration) Calibration of the image is made on the basis of a known distance between the two pixels and their coordinates on the reference image. A calculated ratio enables the measurement of the chip size to an accuracy of $7 \mu \mathrm{m}$.

The purpose of the Image preparation process (Step 3) is to prepare the chip image for the process of image thresholding. The Extract-HSL function changes the 32-bit RGB image to the 8-bit grayscale HSL image. By adjusting brightness, contrast and gamma the differences between the analysed chip and the background are highlighted. By defining the Region of Interest (ROI), the analysed area on the image is determined. Figs. 3a and $3 \mathrm{~b}$ show the image of the captured chip and the effect of image preparation process.

The Image tresholding (Step 4) converts the 8-bit grayscale chip image into a binary image where logic value of all pixels within the selected interval (45-255) is set to 1 . These pixels are treated as white particles or objects of analysis (Fig. 3c). All remaining pixels in the range from 0 to 45 are assigned a value of 0 and the method treats them as black background or noise. These were removed with space filters and filtering process (Step 5). The Space variant Median filter $(3 \times 3)$ is used to smooth the chip shape and to remove the small 
interfering particles. The remaining distracting information on the image is removed by a morphological operator.

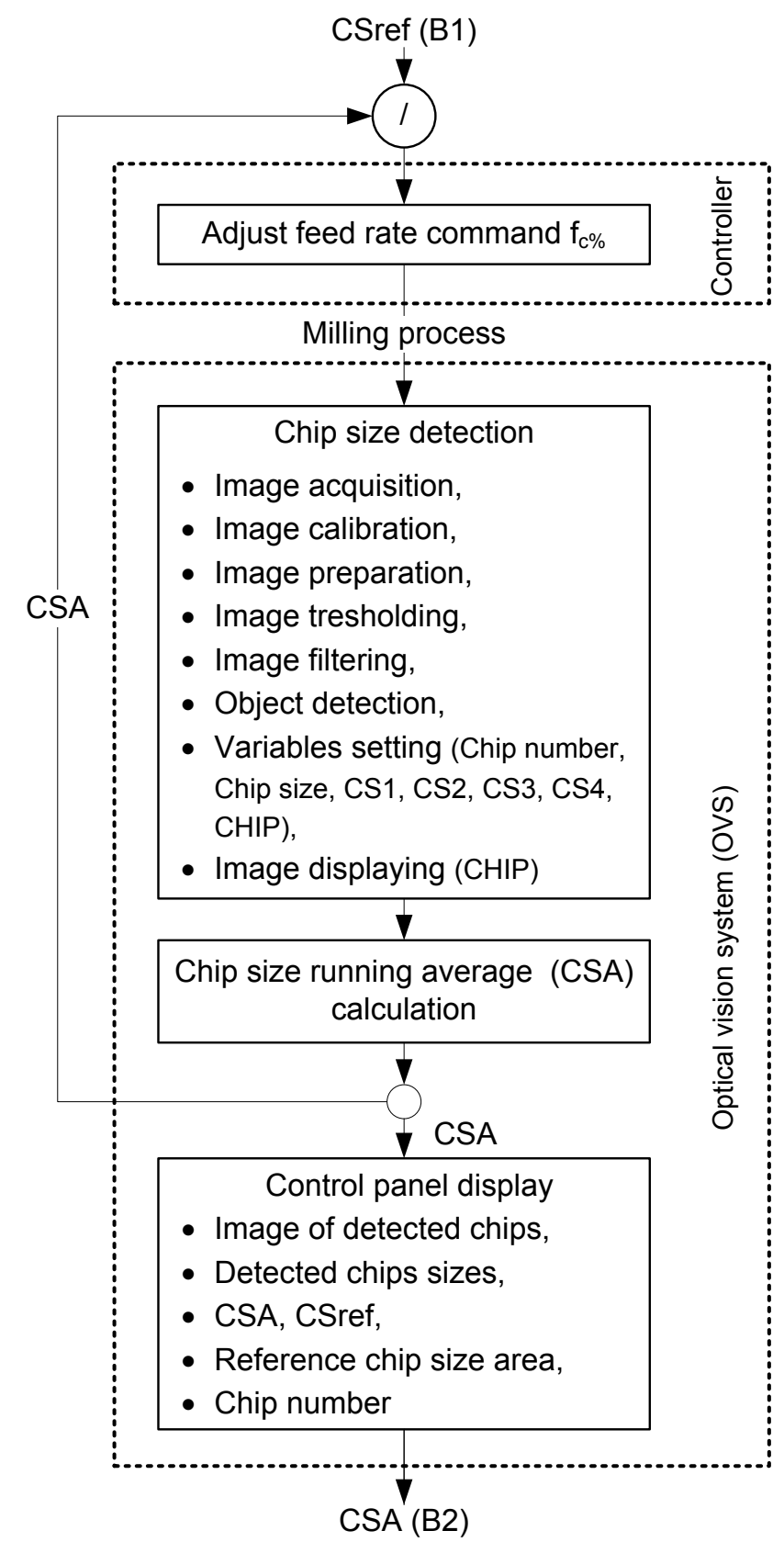

Figure 2: Flow chart of the chip size control algorithm.

Fig. 3a shows the original captured image of the chip after setting the exposure time and gain. Fig. $3 b$ shows the chip image after preparation and Fig. $3 c$ shows the result of tresholding. The arrows indicate the interfering particles that are removed by filtration. After filtration, the image is ready for analysis. In step 6 (object detection), the algorithm locates the objects of homogeneous intensity. The minimal and maximal size of the chip which will be detected is defined. The objects that touch the edges of the region of interest are excluded from analyses and the holes on the detected chips are filled. The detected chips are displayed on a screen, numbered and arranged in order from the largest to the smallest. The chip position, dimensions and surface area (chip size) is determined. 


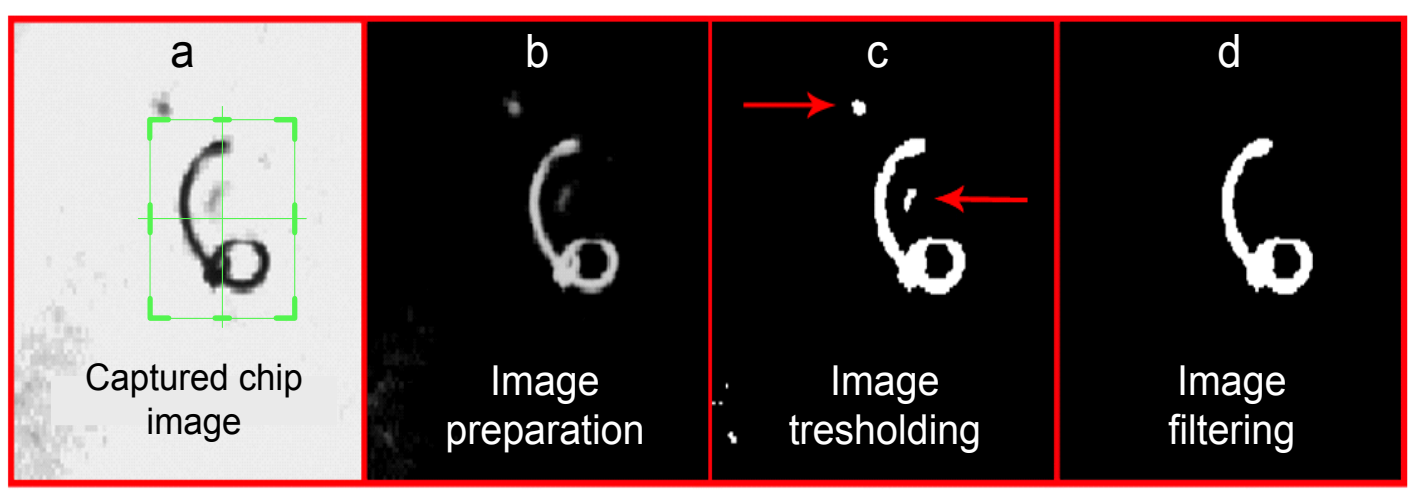

Figure 3: Processing of the detected cutting chip image.

The information transfer between the OVS and the system controller is made through six system variables. These variables created in step 7 are: Chip number, Chip size $\left[\mathrm{mm}^{2}\right], C S 2$ (size of the $2^{\text {nd }}$ largest detected chip) $\left[\mathrm{mm}^{2}\right], C S 3$ (size of the $3^{\text {rd }}$ largest detected chip) $\left[\mathrm{mm}^{2}\right]$, CS4 $\left[\mathrm{mm}^{2}\right]$ and a variable CHIP (image of the detected chip). The variable Chip number contains information on the number of detected chips. The variable Chip size provides information on the size of the largest detected chip. The system variables are linked to many indicators for numerical and graphical display on a control panel of the chip size control system (Fig. 4). In the last step, the program displays the image of the detected chips on the control panel.

\subsection{Calculation of the chip size running average}

In each iteration, the OVS simultaneously detects a large number of chips. The detected chip sizes vary; therefore calculation of the chip size running average is necessary. Calculation of the chip size running average is carried out by shift registers and system variables (Chip size, CS2, CS3, CS4, Chip number). The system variables provide information on the size and the number of detected chips in particular iteration of detection. With the shift registers the sum of the current chip size with the values from the previous iterations is performed. In registry stored new sum of all performed measurements is finally divided by the number of predefined samples (25). The result is the value of the chip size running average (CSA), which is associated with the display indicator on the OVS control panel. The CSA value is transmitted to the system controller.

Fig. 4 shows the control panel of the proposed chip size control system. The limits of the reference chip size area are entered into the system by two graphical sliders in an upper part of the control panel. Two warning indicators inform the operator about the inadequate size of the chip. The OVS button switches between manual and automatic mode of working. An image with the detected chips and a diagram with the values of the CSA are displayed in the middle part of the control panel. The diagram also displays the current size of the detected chip and the allowable area of the chip size. Above the diagram, the user enters the parameter to calculate the $C S A$.

\subsection{System controller design and implementation}

The system controller based on the CSA and determined CSref adjusts the feed rates to ensure optimum machining. The reference model provides information about the CSref. When the $C S A$ exceeds the allowed deviation of the chip size the system with indicators alerts operator of improper feed rates and adjusts them. 


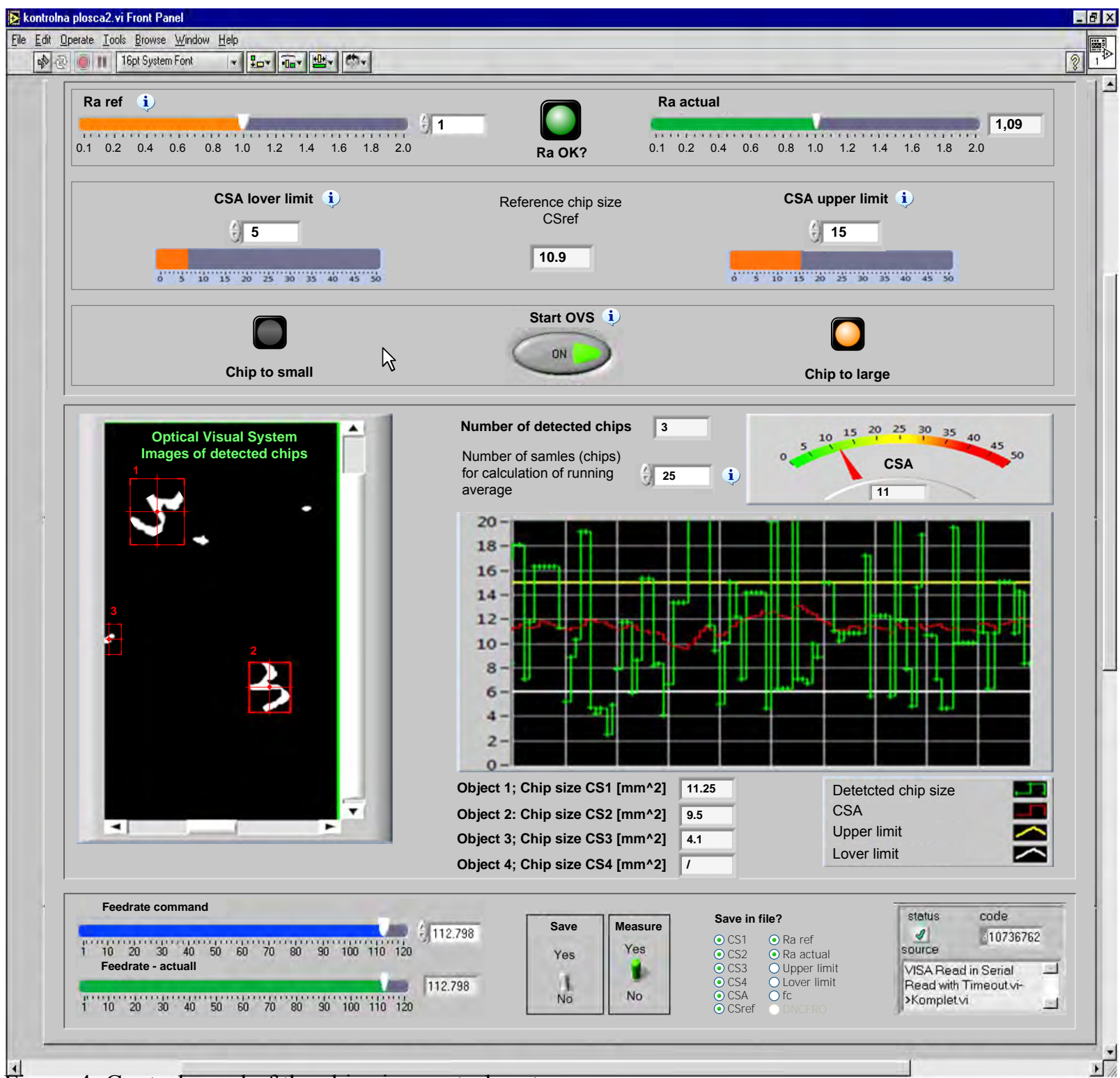

Figure 4: Contro panel of the chip size control system.

In this study two controllers were tested. Their block diagram is presented in Fig. 5. A step controller in $10 \%$ increments decreases or increases the feed rate override percentage until the average size of the chip is not within the desired size range. The step controller has a rapid response, and it is very easy to implement. The second used controller is the division controller. Its structure is a bit more complicated, but still provides fast response times. When adjusting the feed rate it considers a linear correlation between the $C S A$ and the $f_{c \%}$. The new command of feed rate override percentage $f_{c \%}(t)$ is adjusted according to eq. (1):

$$
f_{c \%}(t)=f_{c \%}(t-1) \cdot \frac{C S r e f}{C S A(t)}
$$

In calculating the new commanded feed rate override percentage the previous command of feed rate correction and the ratio between the CSref and the CSA from current iteration is considered.

The stability analysis of the divisional controller is evaluated by simulations with various step changes in the cutter/workpiece contact area. The system is found out to be stable in all the simulation tests, except in cases where the CSA approaches to zero. By limiting the controller commands the problem of the unacceptably large feed rate corrections for very 
small chips is eliminated. By simulations, the optimal value of the controller sample period is determined. Sample period of $110 \mathrm{~ms}$ ensures that the measured value of chip size corresponds with feed rate command from the previous iteration. During all simulation tests the controller manages to return the CSA to the reference chip size within $0.4 \mathrm{~s}$.

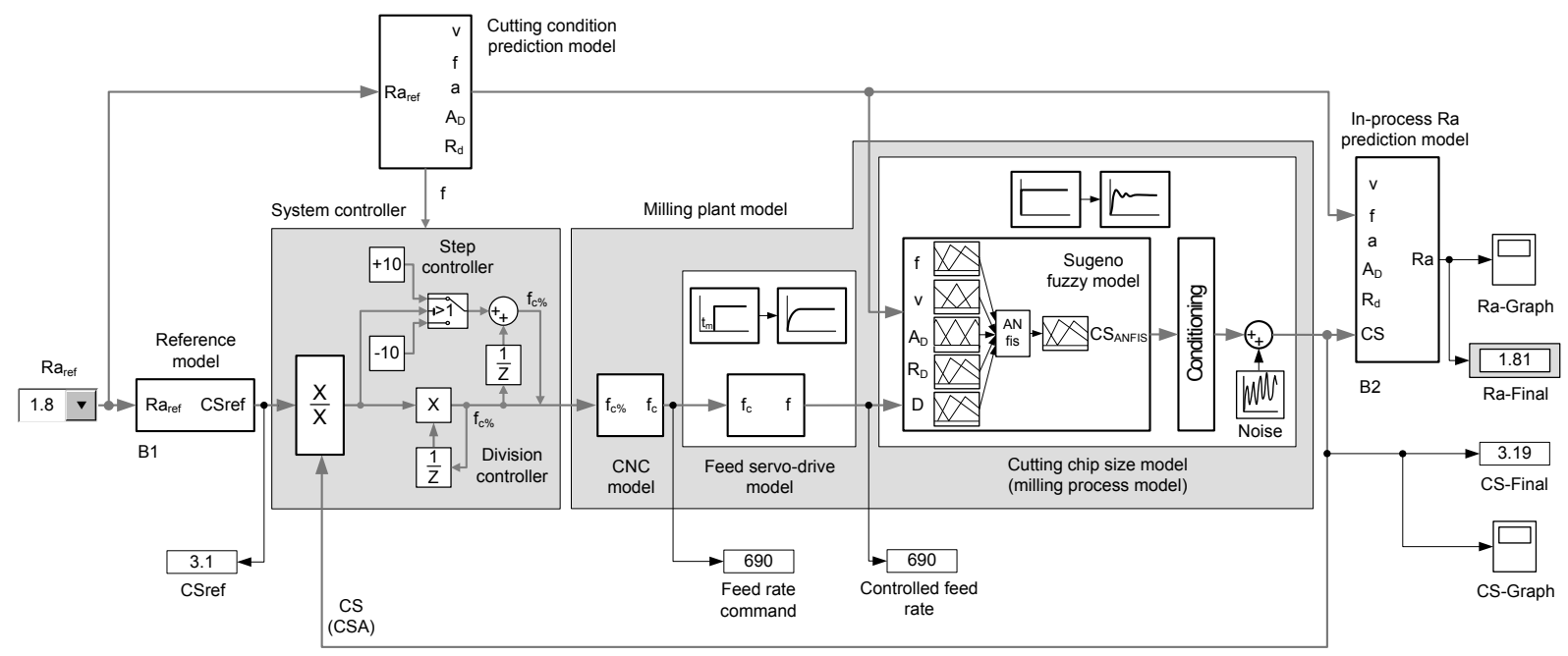

Figure 5: Cutting chip size control simulator.

\section{CHIP SIZE CONTROL SIMULATOR}

The overall simulation block diagram of the proposed visual control system is presented in Fig. 5. It simulates the $R a$ of the machined part by enabling the regulation of chip size. The input to the block diagram is the $R a_{r e f}$ and the output is the simulated $R a$. The control simulator with the corresponding models was developed in Matlab Simulink. Simulator consists of blocks representing the reference model, the system controller, a milling plant model and the surface roughness prediction model. These four elements describe the dynamics of the control simulator.

The main focus of this research was dedicated to the development of the milling plant model. The purpose of this model was to design the chip size controller without expensive and time consuming machining tests. The system controller design is described in the previous chapter. The milling plant model is a feed drive simulator which includes the chip size model (milling process model), servo-control system, a model of CNC controller and chip size conditioning measurements. The adjusted feed rate command is the control signal to the milling plant model and the chip size (CS) is the model output signal.

Fig. 5 outlines the milling plant model. The milling plant can be represented as a product of two transfer functions:

$$
\begin{aligned}
& H_{s}(s)=\frac{f(s)}{f_{c}(s)}=e^{-s t_{m}} \cdot \frac{1}{1+\mathrm{t}_{\mathrm{s}} \cdot s} \\
& H_{p}(s)=\frac{C S(s)}{f(s)}=\frac{C S_{A N F I S}}{f(s)} \cdot \frac{1}{1+\frac{2 \varsigma}{\omega_{n}} \cdot s+\frac{1}{\omega_{n}} \cdot s^{2}}
\end{aligned}
$$

where $C S$ is cutting chip size $\left[\mathrm{mm}^{2}\right], \omega_{n}$ is natural frequency of anti-aliasing filter, $\zeta$ is damping coefficient, $C S_{A N F I S}$ is continuous static signal of the chip size $\left[\mathrm{mm}^{2}\right], f_{c}$ is feed rate command $[\mathrm{mm} / \mathrm{min}], f$ is actual feed rate $[\mathrm{mm} / \mathrm{min}], t_{s}$ is the time constant of the servo control system [s] and $t_{m}$ is the respond time of the servo controls to the commanded feed signal time delay [s]. 
The transfer function $H_{S}$ of the machine dynamics was derived based on the manufacturer's technical data of the machine tool Heller BEA 02 controlled by the Fagor 8040-M CNC system. This approximated transfer function simulates the feed servo-drive response to changes in the commanded feed rate. The appropriate-actual feed rate for a specific cutting situation is provided via the $f_{c \%}$ commands ranging from 0 to $120 \%$.

To verify the dynamic feed servo-drive model defined with $H_{s}$ the experiments were carried out. In step cutting experiments, the input feed rates $(5,10,15$ and $20 \mathrm{~mm} / \mathrm{s})$ were overridden from 0 to $120 \%$. The results showed a good agreement between the experimental and simulation step responses of the feed-drive system. The average time constant $t_{s}$ of the step cutting experiments were measured to be $107 \mathrm{~ms}$ and the difference from the mean values obtained by simulations was less than $4 \%$.

In a first attempt, a mathematical model developed by [20] was used to simulate the size of the cutting chip. In the mathematical model, the chip is restricted by four surfaces; therefore four equations are used to define the chip shape with each equation representing one surface. The Matlab software was used to plot the simulated chip image and to calculate the cutting chip size. The calculated chip size was compared with measured CSA obtained by the vision system. It was found out that the average difference between detected cutting chip size and simulated one is less than $32 \%$. Test results discovered inaccuracies in modelling of the small size chips. Another major disadvantage of mathematical modelling of chip geometry is complex and time-consuming making of the model.

The above mentioned disadvantages were eliminated by applying an Adaptive NeuroFuzzy Inference System (ANFIS) to predict the size of cutting chip in end milling. The ability of ANFIS modelling method to capture the relations between cutting conditions, tool geometry and cutting chip size in an extremely short time has encouraged us to use this method for chip modelling [21]. The method was able to develop the chip size model on-line, during machining in few seconds. This is a non-linear static model, built based on experimental tests. It was incomparably faster than the mathematical model and was easy to implement to the plant model simulator. The prediction error of the trained model is less than $9 \%$.

The basic ANFIS architecture employed to predict the cutting chip size is shown in Fig. 5. The selected fuzzy inference system has 5 inputs and one output. The input set consists of cutting conditions ( 1 - feed rate, 2 - cutting speed, 3, 4 - axial/radial depth of cut) and 5 - the cutting tool diameter $(D)$. The output is cutting chip size $\left(C S_{A N F I S}\right)$. The connections between the inputs and output were first captured during training with a neural network and then expressed in a form of fuzzy rules. A first-order Sugeno fuzzy model with triangular membership functions were selected for training. A combination of a gradient descent method and a least-squares method was selected to tune parameters of the membership functions. The training and testing process was carried out simultaneously during machining tests. The sizes of the chips were measured during machining. Two-thirds of 873 captured data pairs were used for training and the rest for testing. The modelling process was stopped when the prediction testing error has dropped below $9 \%$. Approximately 500 iteration of training was conducted. The 41 fuzzy if-then rules were generated to predict the cutting chip size. The complete milling plant model must also include the conditioning of the optical chip size measurement. This is achieved by a second order low-pass anti-aliasing filter with the natural frequency $\omega_{n}$ and the damping coefficient $\zeta$. Therefore, the milling process and the conditioning transfer function can be expressed according to eq. (3).

The noise model (Fig. 5) introduces a random disturbance into the simulation and ads a greater realism to the simulation. It represents the unmodelled dynamics of the milling process and the chip detection process. The unmodelled dynamics is formed by random values that match with fluctuations of the measured chip size values. 
The simplified model using only the cutting conditions and the cutting chip size is employed to simulate the $R a$. Statistical modelling is employed to provide functional relations between the $R a, C S$, cutting conditions and diameter of the cutter $(D)$. The experimentally obtained surface roughness prediction model is expressed by:

$$
R_{a}=C S^{x_{1}} \cdot f^{x_{2}} \cdot v^{x_{3}} \cdot A_{D}^{x_{4}} \cdot R_{D}^{x_{5}} \cdot D^{x_{6}} \cdot e^{-5.12}
$$

Six coefficients in the model were identified by the pre-process machining tests. The $R a$ is predicted mainly by utilizing the normalized cutting chip size parameter, which is found out to be the most significant. The experimentally determined relations of the $R a$ and the $C S$ have the same trend. The prediction model is obtained at $91 \%$ confident level. Despite of its simplicity, the model is reliable and efficient to predict the $R a$ in-process by utilizing the $C S$.

\section{SIMULATION RESULTS AND DISCUSSION}

The efficiency of the control system is tested by series of simulations under various cutting conditions. They are performed in the presence of random process disturbances simulated by the noise model. The constant chip size control simulation is performed under the variable cutting depths to demonstrate the influence of the variable depth of cut on the $C S$.

The chip size control is evaluated through tree simulation cases: constant cut, step cut and ramp cut. Therefore, the shape of a virtual workpiece is composed of three parts.

The first part of the virtual workpiece (Fig. 6) is designed to analyse crossing of a tool with a constant cutting chip geometry (constant cut, $A_{D}=4 \mathrm{~mm}$ ). The step part allows testing the efficiency of the divisional controller in the presence of a sudden change of the axial depth of cut (step cut $A_{D}=9 \mathrm{~mm}$ ). The ramp part of the workpiece is designed to simulate the linear increasing of cross-section of the chip.

The goal of the simulation is to maintain the $R a_{r e f}$ constant by eliminating the difference between the desired chip size and simulated $C S$. The chip size control simulation is activated by setting the $R a_{r e f}$. The $R a_{r e f}$ for the simulation shown in Fig. 6 is set to $1.8 \mu \mathrm{m}$. An allowed control error is set to be less than $5 \%$ of the $R a_{r e f}$. The CSref is determined according to the block B1. The CSref is set at $3.1 \mathrm{~mm}^{2}$ with $0.3 \mathrm{~mm}^{2}$ of allowed control error.

When the $C S$ is above the upper limit of the allowed cutting chip size control error, the $f_{c \%}$ must be reduced. On the contrary, if the $C S$ is below the lower limit, then the feed rate must be increased.

The initial cutting conditions are determined according to the cutting conditions prediction model (see, Fig. 5). In this model, $16 \mathrm{MnCr} 5$ steel is selected for the work-piece material. The spindle speed is set at $1000 \mathrm{rpm}$, and the axial depth of cut is programmed to undergo step and continuous changes from 4.5 to $9 \mathrm{~mm}$. The radial depth of cut is set to one half of the tool diameter. The simulation is conducted out for a $16 \mathrm{~mm}$ ball end-mill tool equipped with two inserts. The preprogrammed feed rate is set to $850 \mathrm{~mm} / \mathrm{min}$. The feed rate can be overridden from 450 to $1020 \mathrm{~mm} / \mathrm{min}$.

The simulation test is made first without and then with the chip size control action. The final result of the simulation is the value of the $C S$ and the $R a$. The simulation results are shown in Fig. 6.

During virtual machining of the first part of the workpiece (part A-B) without the feed rate adjustment, the $R a$ changes from 1.73 to $1.88 \mu \mathrm{m}$.

Fig. 6a shows the output response signal of the $R a$.

Fig. $6 \mathrm{f}$ is the corresponding response of the $C S$. The $C S$ varies between the value 1.6 and $4.7 \mathrm{~mm}^{2}$. It is evident that the trend of the $C S$ is similar to that of the $R a$ due to the noise model. 


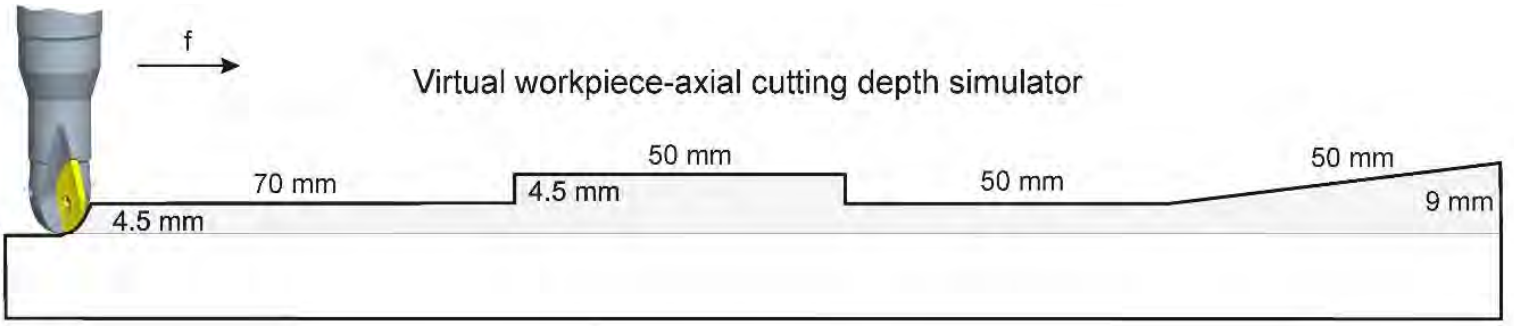

Simulated surface roughness
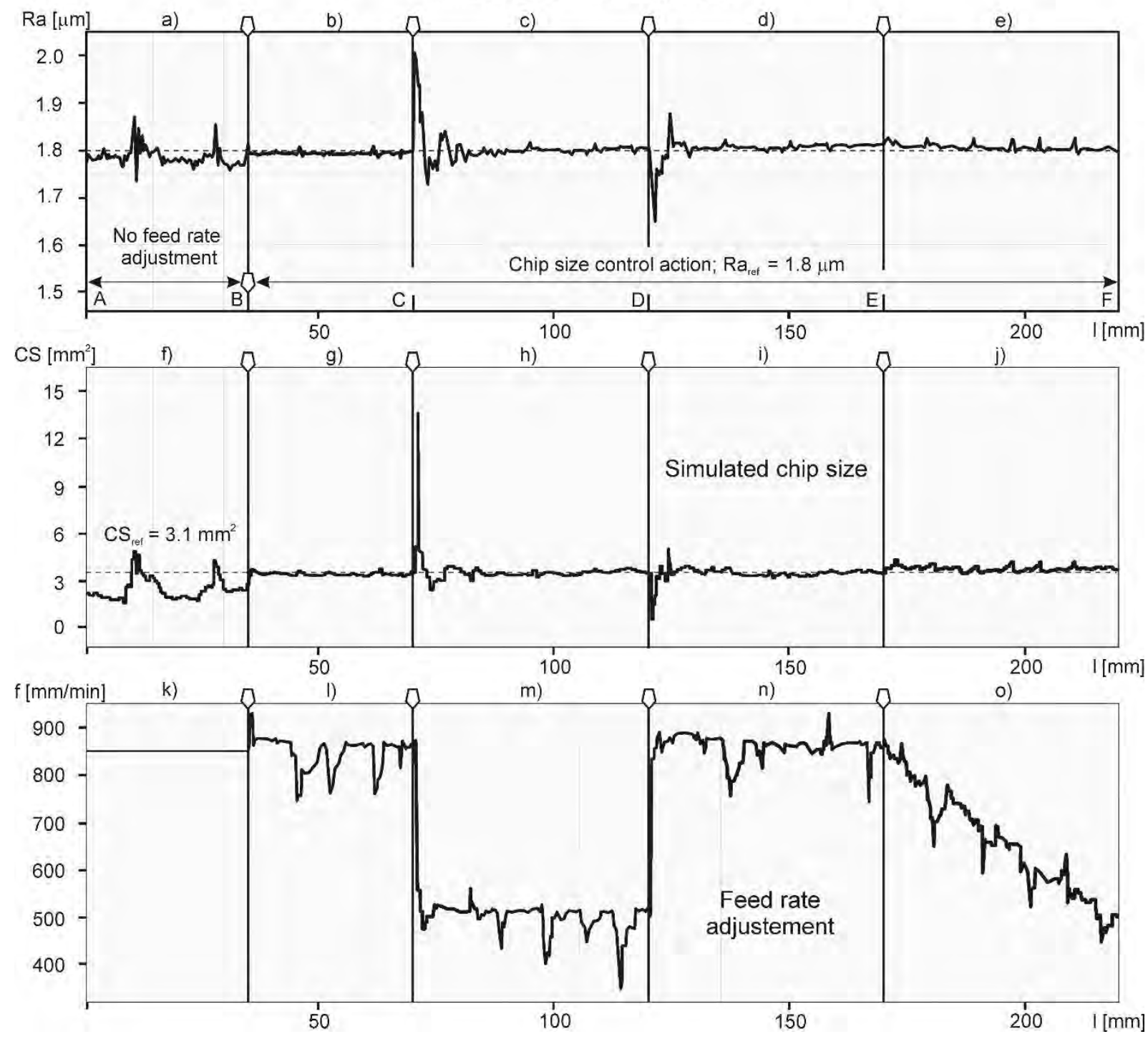

Figure 6: Simulated chip size control response to the various changes in the cutter/workpiece contact area.

The controller is switched on at the position B. First, the controller increases the feed rate up to $930 \mathrm{~mm} / \mathrm{min}$. With the activated chip size control, after the transitional stage, the feed rate is below the feed rate value of $859 \mathrm{~mm} / \mathrm{min}$ for the rest of the part B-C. The $C S$ is constant during virtual machining with $4.5 \mathrm{~mm}$ axial depth of cutting. Figs. $6 \mathrm{f}$, g show the $C S$ variations without and with control action.

The maximum $C S$ is $3.5 \mathrm{~mm}^{2}$ during the pass of the tool from point $\mathrm{B}$ to point $\mathrm{C}$.

At the position $\mathrm{C}$, where the tool encounters the $4.5 \mathrm{~mm}$ step, the feed rate decreases abruptly to maintain the $C S$ at the reference value. In the section of the step part (part C-D), the feed rate drops to the value between 320 and $540 \mathrm{~mm} / \mathrm{min}$ to support the chip size average 
at $3.1 \mathrm{~mm}^{2}$. Fig. $6 \mathrm{~h}$ shows the change of the $C S$. A very large peak of the $C S$ appears when the tool partially encounters the $4.5 \mathrm{~mm}$ step of the virtual workpiece. The controller is able to compensate the induced perturbation. The axial depth of cutting in this section is $9 \mathrm{~mm}$. Fig. 6c shows the output response of the $R a$ on the step variation of the cutting depth by employing the $C S$ control. Fig. $6 \mathrm{~m}$ shows the feed rate response for this case.

At the position E, where the axial depth of cutting drops, the feed rate increases suddenly to keep the $C S$ at the desired range. When the cutting tool starts to leave the step, the $C S$ signal is interrupted by vibrations triggered by a high value of the feed rate. The $C S$ is evolved between 0.5 and $4.2 \mathrm{~mm}^{2}$. When the tool completely leaves the step, the feed rate control signal increases to $861 \mathrm{~mm} / \mathrm{min}$. The upper feed rate limit is set to $1020 \mathrm{~mm} / \mathrm{min}$.

By continuously adjusting of feed rate in the segment of the ramp part (part D-E), the $C S$ is maintained constant so that the $R a_{r e f}$ can be reached. As the $A_{D}$ increases continuously, the feed rates decrease accordingly and thus maintaining the $R a$ at the reference value. The variation of axial depth of cut is accurately compensated. The simulation demonstrates that $R a$ can be maintained constant by controlling the cutting chip size.

\section{CONCLUSION}

In this paper, the visual control of cutting chip size is employed to maintain the surface roughness constant during ball-end milling. The efficiency of the chip size control strategy is tested by a series of simulations. The stability analysis of the system controller is evaluated by simulations with various step changes in the cutter/workpiece contact area. The milling plant model was developed by integrating the $\mathrm{CNC}$ model, the feed servo dive model and the cutting chip size model. The study's main findings are summarized below:

- The cutting chips were timely detected by the optical vision system.

- The chip size running average (CSA) was successfully used to control the machined Ra.

- Statistical modelling is successfully employed to provide functional relations between $R a$, $C S$ and cutting conditions. The statistical model is economical and more efficient than the existing in-process surface roughness measurement techniques.

- The ANFIS method is employed to predict the cutting chip size in ball-end milling. The ANFIS model was much faster than the mathematical model and was easy to implement to the milling plant model simulator.

- The simulation results show that the applied division controller is efficient and stable under variable cutting conditions that are typical for ball-end milling.

The proposed visual control of the cutting chip geometry provides a novel way for modelling and control of surface roughness in machining processes.

\section{REFERENCES}

[1] Al-Kindi, G. A.; Shirinzadeh, B. (2007). An evaluation of surface roughness parameters measurement using vision-based data, International Journal of Machine Tools and Manufacture, Vol. 47, No. 3-4, 697-708, doi:10.1016/j.ijmachtools.2006.04.013

[2] Lee, B. Y.; Tarng, Y. S. (2001). Surface roughness inspection by computer vision in turning operations, International Journal of Machine Tools and Manufacture, Vol. 41, No. 9, 1251-1263, doi:10.1016/S0890-6955(01)00023-2

[3] Young, R. D.; Vorburger, T. V.; Teague, E. C. (1980). In-process and on-line measurement of surface finish, CIRP Annals - Manufacturing Technology, Vol. 29, No. 1, 435-440, doi:10.1016/ $\underline{\text { S0007-8506(07)61366-3 }}$

[4] Sundar, S. M.; Raman, S. (1993). Analysis for vision assisted optical characterization of machined surfaces, Proceedings of the 1993 ASME Winter Annual Meeting, New Orleans, Vol. 64, $43-58$ 
[5] Kamguem, R.; Tahan, S. A.; Songmene, V. (2013). Evaluation of machined part surface roughness using image texture gradient factor, International Journal of Precision Engineering and Manufacturing, Vol. 14, No. 2, 183-190, doi:10.1007/s12541-013-0026-x

[6] Lu, R.-S.; Tian, G. Y. (2006). Online measurement of surface roughness by laser light scattering, Measurement Science and Technology, Vol. 17, No. 6, 1496-1502, doi:10.1088/0957$\underline{0233 / 17 / 6 / 030}$

[7] Garbini, J. L.; Koh, S.-P.; Jorgensen, J. E.; Ramulu, M. (1992). Surface profile measurement during turning using fringe-field capacitive profilometry, Journal of Dynamic Systems, Measurement, and Control, Vol. 114, No. 2, 234-243, doi:10.1115/1.2896520

[8] Shin, Y. C.; Oh, S. J.; Coker, S. A. (1995). Surface roughness measurement by ultrasonic sensing for in-process monitoring, Journal of Engineering for Industry, Vol. 117, No. 3, 439-447, doi: $10.1115 / 1.2804352$

[9] Coker, S. A.; Shin, Y. C. (1996). In-process control of surface roughness due to tool wear using a new ultrasonic system, International Journal of Machine Tools and Manufacture, Vol. 36, No. 3, 411-422, doi:10.1016/0890-6955(95)00057-7

[10] Karayel, D. (2009). Prediction and control of surface roughness in CNC lathe using artificial neural network, Journal of Materials Processing Technology, Vol. 209, No. 7, 3125-3137, doi:10.1016/j.jmatprotec.2008.07.023

[11] Lee, B. Y.; Yu, S. F.; Juan, H. (2004). The model of surface roughness inspection by vision system in turning, Mechatronics, Vol. 14, No. 1, 129-141, doi:10.1016/S0957-4158(02)00096-X

[12] Ho, S.-Y.; Lee, K.-C.; Chen, S.-S.; Ho, S.-J. (2002). Accurate modeling and prediction of surface roughness by computer vision in turning operations using an adaptive neuro-fuzzy inference system, International Journal of Machine Tools and Manufacture, Vol. 42, No. 13, 1441-1446, doi:10.1016/S0890-6955(02)00078-0

[13] Shiraishi, M.; Sato, S. (1990). Dimensional and surface roughness controls in a turning operation, Journal of Engineering for Industry, Vol. 112, No. 1, 78-83, doi:10.1115/1.2899298

[14] Tsai, Y.-H.; Chen, J. C.; Lou, S.-J. (1999). An in-process surface recognition system based on neural networks in end milling cutting operations, International Journal of Machine Tools and Manufacture, Vol. 39, No. 4, 583-605, doi:10.1016/S0890-6955(98)00053-4

[15] Simunovic, G.; Simunovic, K.; Saric, T. (2013). Modelling and simulation of surface roughness in face milling, International Journal of Simulation Modelling, Vol. 12, No. 3, 141-153, doi:10.2507/IJSIMM12(3)1.219

[16] Huang, B. P.; Chen, J. C.; Li, Y. (2008). Artificial-neural-networks-based surface roughness Pokayoke system for end-milling operations, Neurocomputing, Vol. 71, No. 4-6, 544-549, doi:10.1016/j.neucom.2007.07.029

[17] Kirby, E. D.; Chen, J. C.; Zhang, J. Z. (2006). Development of a fuzzy-nets-based in-process surface roughness adaptive control system in turning operations, Expert Systems with Applications, Vol. 30, No. 4, 592-604, doi:10.1016/j.eswa.2005.07.005

[18] Cus, F.; Zuperl, U. (2013). Control strategy for assuring constant surface finish by controlling cutting forces, Transactions of FAMENA, Vol. 37, No. 3, 41-52

[19] Cus, F.; Zuperl, U. (2015). Surface roughness control simulation of turning processes, Strojniski vestnik - Journal of Mechanical Engineering, Vol. 61, No. 4, 245-253, doi:10.5545/svjme.2014.2345

[20] Zhang, G.; To, S. (2015) A novel surface quality evaluation method in ultra-precision raster milling using cutting chips, Journal of Materials Processing Technology, Vol. 219, 328-338, doi:10.1016/j.jmatprotec.2014.10.010

[21] Cus, F.; Zuperl, U. (2011). Merged neural decision system and ANFIS wear prediction for supporting tool condition monitoring, Transactions of FAMENA, Vol. 35, No. 1, 13-26 